\title{
Searching for $\mathbf{H}_{2}$ emission from protoplanetary disks using near- and mid-infrared high-resolution spectroscopy
}

\author{
A. Carmona ${ }^{1,2,3} \dagger$, M. E. van den Ancker ${ }^{3}$, Th. Henning ${ }^{2}$, \\ Ya. Pavlyuchenkov ${ }^{2}$, C. P. Dullemond ${ }^{2}$, M. Goto ${ }^{2}$, D. Fedele Fed, $^{2,3,}$, \\ B. Stecklum ${ }^{5}$, W. F-.Thi ${ }^{6}$, J. Bouwman ${ }^{2}$ and L. B. F. M. Waters ${ }^{7,8}$ \\ ${ }^{1}$ ISDC \& Geneva Observatory, University of Geneva, \\ chemin d'Ecogia 16, CH-1290 Versoix. Switzerland \\ email:Andres.Carmona@obs.unige.ch \\ ${ }^{2}$ Max Planck Institute for Astronomy, \\ Königstuhl 17, 69117 Heidelberg, Germany \\ ${ }^{3}$ European Southern Observatory, \\ Karl Schwarzschild Strasse 2,85748 Garching bei München, Germany \\ ${ }^{4}$ Dipartimento di Astronomia, Università di Padova, \\ Vicolo dell'Osservatorio 2, 35122 Padova, Italy \\ ${ }^{5}$ Thüringer Landessternwarte Tautenburg, \\ Sternwarte 5, 07778 Tautenburg, Germany \\ ${ }^{6}$ Royal Observatory Edinburgh, \\ Blackford Hill, Edinburgh, EH9 3HJ, UK \\ ${ }^{7}$ Astronomical Institute, University of Amsterdam, \\ Kruislaan 403, NL-1098 SJ Amsterdam, The Netherlands, \\ ${ }^{8}$ Instituut voor Sterrenkunde, Katholieke Universiteit \\ Leuven, Celestijnenlaan 200B, B-3030 Heverlee, Belgium
}

\begin{abstract}
The mass and dynamics of protoplanetary disks are dominated by molecular hydrogen $\left(\mathrm{H}_{2}\right)$. However, observationally very little is known about the $\mathrm{H}_{2}$. In this paper, we discuss two projects aimed to constrain the properties of $\mathrm{H}_{2}$ in the disk's planet forming region $(\mathrm{R}<50 \mathrm{AU})$. First, we present a sensitive survey for pure-rotational $\mathrm{H}_{2}$ emission at 12.278 and $17.035 \mu \mathrm{m}$ in a sample of nearby Herbig Ae/Be and T Tauri stars using VISIR, ESO's VLT high-resolution mid-infrared spectrograph. Second, we report on a search for $\mathrm{H}_{2}$ ro-vibrational emission at 2.1228, 2.2233 and $2.2477 \mu \mathrm{m}$ in the classical T Tauri star $\mathrm{LkH} \alpha 264$ and the debris disk 49 Cet employing CRIRES, ESO's VLT high-resolution near-infrared spectrograph.

VISIR project: none of the sources show $\mathrm{H}_{2}$ mid-IR emission. The observed disks contain less than a few tenths of $\mathrm{M}_{\mathrm{Jupiter}}$ of optically thin $\mathrm{H}_{2}$ at $150 \mathrm{~K}$, and less than a few $\mathrm{M}_{\text {Earth }}$ at $T>300 \mathrm{~K}$. Our non-detections are consistent with the low flux levels expected from the small amount of $\mathrm{H}_{2}$ gas in the surface layer of a Chiang and Goldreich (1997) Herbig Ae two-layer disk model. In our sources the $\mathrm{H}_{2}$ and dust in the surface layer have not significantly departed from thermal coupling $\left(\mathrm{T}_{\mathrm{gas}} / \mathrm{T}_{\mathrm{dust}}<2\right)$ and the gas-to-dust ratio in the surface layer is very likely $<1000$.

CRIRES project: The $\mathrm{H}_{2}$ lines at $2.1218 \mu \mathrm{m}$ and $2.2233 \mu \mathrm{m}$ are detected in $\mathrm{LkH} \alpha 264$. An upper limit on the $2.2477 \mu \mathrm{m} \mathrm{H}_{2}$ line flux in $\mathrm{LkH} \alpha 264$ is derived. 49 Cet does not exhibit $\mathrm{H}_{2}$ emission in any of observed lines. There are a few $\mathrm{M}_{\mathrm{M} \text { oon }}$ of optically thin hot $\mathrm{H}_{2}$ in the inner disk ( $\sim 0.1 \mathrm{AU})$ of $\mathrm{LkH} \alpha 264$, and less than a tenth of a $\mathrm{M}_{\mathrm{M} \text { oon }}$ of hot $\mathrm{H}_{2}$ in the inner disk of 49 Cet. The shape of the 1-0 S(0) line indicates that $\mathrm{LkH} \alpha$ disk is close to face-on $\left(i<35^{\circ}\right)$. The measured 1-0 S(0)/1-0 S(1) and 2-1 S(1)/1-0 S(1) line ratios in LkH $\alpha 264$ indicate that the $\mathrm{H}_{2}$ is thermally excited at $T<1500 \mathrm{~K}$. The lack of $\mathrm{H}_{2}$ emission in the NIR spectra of 49 Cet and the absence of $\mathrm{H} \alpha$ emission suggest that the gas in the inner disk of 49 Cet has dissipated.
\end{abstract}

$\dagger$ This paper is based on materials of our A\&A papers Carmona et al. 2008 \& 2007. 
Keywords. stars: emission-line - stars: pre-main sequence - planetary systems:protoplanetary disks

\section{Introduction}

Circumstellar disks surrounding low- and intermediate- mass stars in their pre-main sequence phase are the locations where planets presumably form. Such protoplanetary disks are composed of gas and dust. Their mass and dynamics are dominated by gas $(99 \%)$, specifically by molecular hydrogen $\left(\mathrm{H}_{2}\right)$. Observationally, very little is known about the gas compared to the dust. However, if we want to answer major questions in planet formation such as: how massive are the disks?, how extended are the disks?, and how long protoplanetary disks last? we require information about the gaseous component of the disk. In particular, we would like to characterize warm gas in the inner disk $(\mathrm{R}<$ $50 \mathrm{AU}$ ), the region where planets form (see reviews by Najita et al. 2007; Carr 2005).

$\mathrm{H}_{2}$ is by far the most abundant molecular species in protoplanetary disks. Unfortunately, $\mathrm{H}_{2}$ is one of the most challenging molecules to detect. $\mathrm{H}_{2}$ is a homonuclear molecule that lacks a permanent dipole moment, therefore, $\mathrm{H}_{2}$ transitions are electric quadrupole in nature, thus, very weak (i.e., small Einstein coefficients). In addition, in the case of protoplanetary disks, the $\mathrm{H}_{2}$ lines are not sensitive to the gas in the optically thick regions where the dust and gas are at equal temperature. Practical observational challenges also have to be faced. The $\mathrm{H}_{2}$ lines from the disk needs to be detected on the top of a strong infrared continuum. From the ground, the infrared windows (specially the mid-infrared) are strongly affected by sky and instrument background emission, and the $\mathrm{H}_{2}$ transitions lie close to atmospheric absorption lines highly dependent on atmospheric conditions. The advent of high spectral resolution infrared spectrographs mounted on large aperture telescopes, allows for the first time the study of $\mathrm{H}_{2}$ emission from the ground.

In this paper, we discuss two projects aimed to constrain the properties of $\mathrm{H}_{2}$ gas in the planet forming region of circumstellar disks. In Sect. 1 we present the results of a large, sensitive survey for pure-rotational molecular hydrogen emission at 12.278 and 17.035 microns in a sample of nearby Herbig Ae/Be and T Tauri stars using the high-resolution mid-infrared spectrograph VISIR at ESO-VLT (Carmona et al. 2008). In Sect. 2. we report on the results of a search for $\mathrm{H}_{2}$ ro-vibrational emission at 2.1228, 2.2233 and $2.2477 \mu \mathrm{m}$ in the classical T Tauri star $\mathrm{LkH} \alpha 264$ and the debris disk 49 Cet using CRIRES, the new ESO's VLT Adaptive Optics high resolution near-infrared spectrograph (Carmona et al. 2007).

\section{Searching for mid-IR $\mathbf{H}_{2}$ emission from protoplanetary disks with VISIR}

\subsection{Motivation.}

To probe the gas in the giant planet forming region of the disk. $\mathrm{H}_{2}$ mid-IR lines probe warm gas at $T \sim 150-1000 \mathrm{~K}$. This gas is located from a few AU up to $50 \mathrm{AU}$.

\subsection{Previous work.}

$\mathrm{H}_{2}$ mid-IR emission from protoplanetary disks has been reported from ISO observations (Thi et al. 2001). However, subsequent ground-based efforts (Richter et al. 2002; Sheret et al. 2003; Sako et al. 2005) did not confirm the ISO detections. $\mathrm{H}_{2}$ emission in the mid-IR has been searched towards debris disks using Spitzer (Hollenbach et al. 2005, Pascucci et al. 2006, Chen et al. 2006) with no detection reported. Most recently, Bitner et al. (2007) and Martin-Zaïdi et al. (2007) reported the detection of mid-IR $\mathrm{H}_{2}$ emission in two Herbig Ae/Be stars (AB Aur and HD 97048) from the ground, and Lahuis et al. (2007) reported the detection of mid-IR $\mathrm{H}_{2}$ emission in 6 T Tauri stars with Spitzer. 

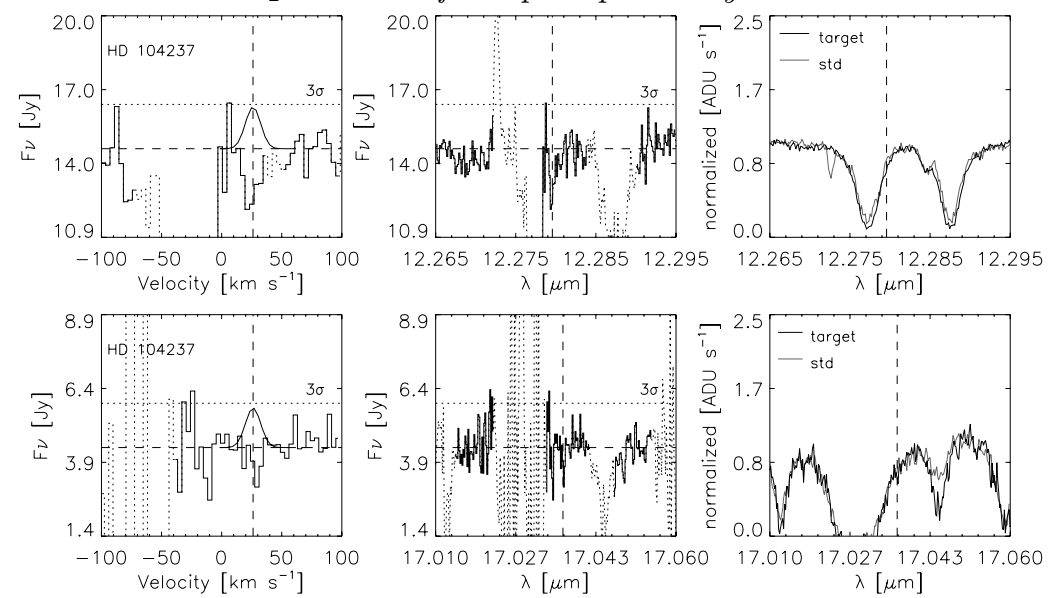

Figure 1. Example of the VISIR spectra obtained in the case of HD 104237. The upper panels show the spectra for the $\mathrm{H}_{2} \quad 0-0 \mathrm{~S}(2)$ line at $12.278 \mu \mathrm{m}$. The lower panels display the spectra for the $\mathrm{H}_{2} 0-0 \mathrm{~S}(1)$ line at $17.035 \mu \mathrm{m}$. The left panels show a zoom to the -100 to $100 \mathrm{~km} \mathrm{~s}^{-1}$ interval of the atmospheric corrected spectra. A Gaussian of $F W H M=15 \mathrm{~km} \mathrm{~s}^{-1}$ and integrated line flux equal to the line-flux upper limits obtained is overplotted at the expected velocity shifted location (vertical dashed line). The central panels show the full corrected spectra. Dotted lines show spectral regions strongly affected by telluric or standard star absorption features. The right panels show the continuum normalized spectra of the standard star and the target before telluric correction. The spectra are not corrected for the radial velocity of the targets (Carmona et al. 2008).

\subsection{Observations \& Data Reduction.}

We observed the Herbig Ae/Be stars UX Ori, HD 34282, HD 100453, HD 101412, HD 104237 and HD 142666, and the T Tauri star HD 319139 in the first semester of 2006 and 2007 with VISIR, a combined imager and spectrograph designed for observations in the $N(\approx 8-13 \mu \mathrm{m})$ and $Q$ bands $(\approx 16.5-24.5 \mu \mathrm{m})$ (Lagage et al. 2004), mounted at the ESO-VLT Melipal telescope in Cerro Paranal, Chile. We selected a sample of well known nearby Herbig Ae/Be and T Tauri stars based on evidence of large disk reservoirs. The targets have either reported detections of cold CO gas at (sub)-mm wavelengths, or dust continuum emission at mm wavelengths. We chose stars with $12 \mu \mathrm{m}$ continuum fluxes $>0.5 \mathrm{Jy}$ (otherwise too faint for acquisition with VISIR) and $<25 \mathrm{Jy}$ (hard to detect weak lines on top of a strong continuum). The $\mathrm{H}_{2} v=0-0 S(1)$ line at $17.035 \mu \mathrm{m}$ was observed in the high-resolution long-slit mode with a 0.4 arcsec slit, giving a spectral resolution $\mathrm{R} \approx 21000$, or $14 \mathrm{~km} \mathrm{~s}^{-1}$. The $\mathrm{H}_{2} v=0-0 S(2)$ line at $12.278 \mu \mathrm{m}$ was observed in the high-resolution echelle mode with a 0.4 arcsec slit, giving a spectral resolution $\mathrm{R}$ $\approx 20000$, or $15 \mathrm{~km} \mathrm{~s}^{-1}$. The total integration time in each line was $1 \mathrm{~h}$. The slit was oriented in the North-South direction. Sky background was subtracted by chopping the telescope by $\sim 8^{\prime \prime}$ in the direction of the slit. Asymmetrical thermal background of the telescope was subtracted by nodding the telescope by $\sim 8^{\prime \prime}$ in the direction of the slit.

For correcting the spectra for telluric absorption and obtaining the absolute flux calibration, spectroscopic standard stars at close airmasses to that of the science targets were observed immediately preceding and following the $12 \mu \mathrm{m}$ exposure, and preceding or following the $17 \mu \mathrm{m}$ exposure. After assuring that all the half-chop cycles in the VISIR data cubes had the same wavelength in the same row of pixels, the data-cubes were processed with the VISIR pipeline (Lundin 2006). The science spectrum was extracted by summing the number of counts inside the PSF in the dispersion direction in the 2D spectrum. To correct for telluric absorption and flux-calibrate the science spectrum, the onedimensional extracted science spectrum was divided by the one-dimensional extracted 
Table 1. $\mathrm{H}_{2}$ mid-IR emission line flux upper limits and optically thin $\mathrm{H}_{2}$ gas mass limits.

\begin{tabular}{|c|c|c|c|c|c|c|c|c|}
\hline Star & $\mathrm{H}_{2}$ line & $\begin{array}{c}\lambda \\
{[\mu \mathrm{m}]}\end{array}$ & $\begin{array}{c}\text { continuum } \\
{[\mathrm{Jy}]}\end{array}$ & \multicolumn{2}{|c|}{$\left[\begin{array}{c}\text { line flux } \\
{\left[\times 10^{-14} \operatorname{ergs~s}^{-1} \mathrm{~cm}^{-2}\right]}\end{array}\right.$} & $T=150 \mathrm{~K}$ & $\mathrm{H}_{2}$ mass limits in $\mathrm{M}_{J}$ & $\begin{array}{l}\mathrm{n} \mathrm{M}_{J} \\
\quad T=1000 \mathrm{~K}\end{array}$ \\
\hline \multirow[t]{2}{*}{ UX Ori } & $0-0 \mathrm{~S}(2)$ & 12.278 & $1.9(1.1)$ & & $<1.4$ & 27.9 & $1.9 \times 10^{-1}$ & $1.2 \times 10^{-2}$ \\
\hline & $0-0 \mathrm{~S}(1)$ & 17.035 & $2.0(1.5)$ & & $<1.3$ & 1.0 & $6.8 \times 10^{-2}$ & $2.0 \times 10^{-2}$ \\
\hline \multirow[t]{2}{*}{ HD 34282} & $0-0 \mathrm{~S}(2)$ & 12.278 & $0.3(0.4)$ & & $<0.5$ & 13.8 & $9.7 \times 10^{-2}$ & $6.0 \times 10^{-3}$ \\
\hline & $0-0 \mathrm{~S}(1)$ & 17.035 & $\ldots$ & & $\ldots$ & $\ldots$ & & 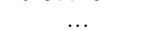 \\
\hline \multirow[t]{2}{*}{ HD 100453} & $0-0 \mathrm{~S}(2)$ & 12.278 & $9.0(0.7)$ & & $<0.9$ & 1.9 & $1.4 \times 10^{-2}$ & $0.9 \times 10^{-3}$ \\
\hline & $0-0 \mathrm{~S}(1)$ & 17.035 & $14.8(1.7)$ & & $<1.5$ & 0.1 & $0.9 \times 10^{-2}$ & $2.5 \times 10^{-3}$ \\
\hline \multirow[t]{2}{*}{ HD 101412} & $0-0 \mathrm{~S}(2)$ & 12.278 & $3.5(1.0)$ & & $<1.2$ & 5.3 & $3.7 \times 10^{-2}$ & $2.3 \times 10^{-3}$ \\
\hline & $0-0 \mathrm{~S}(1)$ & 17.035 & $1.6(1.6)$ & & $<1.4$ & 0.2 & $1.6 \times 10^{-2}$ & $4.8 \times 10^{-3}$ \\
\hline \multirow[t]{2}{*}{ HD 104237} & $0-0 \mathrm{~S}(2)$ & 12.278 & $14.6(1.8)$ & & $<2.2$ & 5.0 & $3.6 \times 10^{-2}$ & $2.2 \times 10^{-3}$ \\
\hline & $0-0 \mathrm{~S}(1)$ & 17.035 & $4.4(1.5)$ & & $<1.3$ & 0.1 & $0.8 \times 10^{-2}$ & $2.3 \times 10^{-3}$ \\
\hline \multirow[t]{2}{*}{ HD 142666} & $0-0 \mathrm{~S}(2)$ & 12.278 & $\ldots$ & & $\ldots$ & $\ldots$ & $\ldots$ & $\ldots$ \\
\hline & $0-0 \mathrm{~S}(1)$ & 17.035 & $8.0(1.2)$ & & $<1.1$ & 0.2 & $1.0 \times 10^{-2}$ & $3.1 \times 10^{-3}$ \\
\hline \multirow[t]{2}{*}{ HD 319139} & $0-0 \mathrm{~S}(2)$ & 12.278 & $\ldots$ & & $\ldots$ & $\ldots$ & $\ldots$ & $\ldots$ \\
\hline & $0-0 \mathrm{~S}(1)$ & 17.035 & $2.5(1.5)$ & & $<1.3$ & 0.2 & $1.2 \times 10^{-2}$ & $3.7 \times 10^{-3}$ \\
\hline
\end{tabular}

Notes: ${ }^{a}$ Upper limits calculated using a line $F W H M$ of $15 \mathrm{~km} \mathrm{~s}^{-1}$.

spectrum of the standard star (for further details on the data reduction procedure, telluric correction and flux calibration see Sect. 3 of Carmona et al. 2008).

\subsection{Results.}

None of the observed sources show evidence for $\mathrm{H}_{2}$ emission at 12 or $17 \mu \mathrm{m}$ (see Fig. 1 for an example of the spectra obtained in the case of HD 104237, for all the spectra see Carmona et al. 2008). Assuming that the $\mathrm{H}_{2}$ lines are unresolved, we calculated $3 \sigma$ upper limits to the integrated $\mathrm{H}_{2}$ line fluxes by multiplying the $3 \sigma$ continuum flux noise with the instrument resolution line width $\left(\sim 15 \mathrm{~km} \mathrm{~s}^{-1}\right)$. Our results are summarized in Table 1. The typical sensitivity limit of our observations is a line flux of $10^{-14} \mathrm{ergs} \mathrm{s}^{-1} \mathrm{~cm}^{-2}$. Our flux limits are of the order of magnitude of the $\mathrm{H}_{2} 0-0 \mathrm{~S}(1)$ and $0-0 \mathrm{~S}(2)$ lines fluxes (1.1 and $0.53 \times 10^{-14} \mathrm{ergs} \mathrm{s}^{-1} \mathrm{~cm}^{-2}$, respectively) reported by Bitner et al. (2007) for $\mathrm{AB}$ Aur $\dagger$ and the $\mathrm{H}_{2}$ line fluxes of 0.33 to $1.70 \times 10^{-14} \mathrm{ergs} \mathrm{s}^{-1} \mathrm{~cm}^{-2}$ reported for the $\mathrm{H}_{2}$ 0-0 S(2) line in the observations by Lahuis et al. (2007).

\subsection{Discussion.}

Under the assumption that the $\mathrm{H}_{2}$ emission and the emission of the accompanying dust are optically thin, that the emitting $\mathrm{H}_{2}$ is in local thermodynamical equilibrium (LTE), and that the source size is equal or smaller than VISIR's beam size, we derived upper limits to the $\mathrm{H}_{2}$ mass as a function of the temperature employing (Thi et al. 2001)

$$
M_{\text {gas }}=f \times 1.76 \times 10^{-20} \frac{4 \pi d^{2} F_{u l}}{E_{u l} A_{u l} x_{u}(T)} M_{\odot},
$$

where $F_{u l}$ is the upper limit to the integrated line flux, $d$ is the distance in pc to the star, $E_{u l}$ is the energy of the transition, $A_{u l}$ is the Einstein coefficient of the $J=u-l$ transition and $x_{u}$ is the population of the level $u$ at the excitation temperature $T$ in LTE; $f$ is the conversion factor required for deriving the total gas mass from the $\mathrm{H}_{2}$-ortho or $\mathrm{H}_{2}$-para mass determined. In Table 1, we present our results. The disks contain less than a few tenths of Jupiter mass of optically thin $\mathrm{H}_{2}$ at $150 \mathrm{~K}$, and less than a few Earth masses of optically thin $\mathrm{H}_{2}$ at $300 \mathrm{~K}$ and higher temperatures.

Using a two-layer Chiang and Goldreich (1997, CG 97) disk model implementation (CGplus, Dullemond et al. 2001) with physical parameters aimed to fit the spectral

$\dagger$ Note that the observations of AB Aur were performed with TEXES, which provides a spectral resolution of 100000 , increasing the line-to-continuum contrast compared with our observations. 


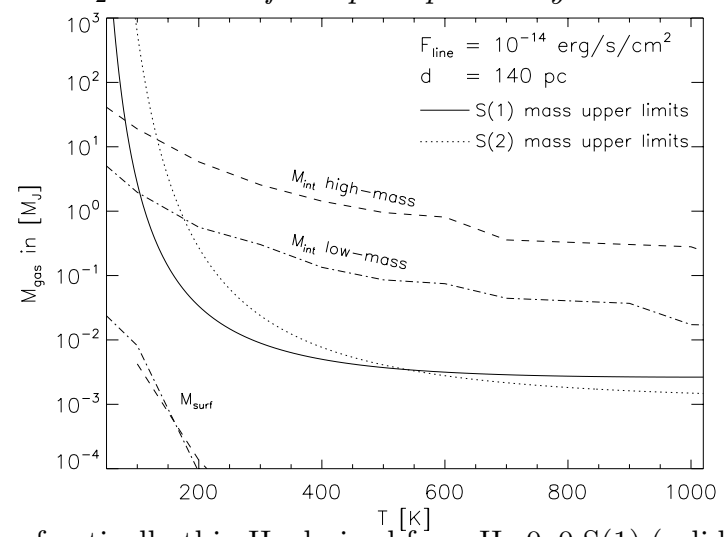

Figure 2. Mass limits of optically thin $\mathrm{H}_{2}$ derived from $\mathrm{H}_{2} 0-0 \mathrm{~S}(1)$ (solid line) and $\mathrm{H}_{2} 0-0 \mathrm{~S}(2)$ (dotted line) as a function of the temperature for a line flux limit of $10^{-14} \mathrm{ergs} \mathrm{s}^{-1} \mathrm{~cm}^{-2}$ for a source at a distance of 140 pc. Dashed and dot-dashed lines show the gas mass as function of the temperature for a Chiang and Goldreich (1997) optically thick two-layer model for a low-mass $\left(M_{\mathrm{DISK}}=0.02 M_{\odot}\right)$ and a high-mass $\left(M_{\mathrm{DISK}}=0.11 M_{\odot}\right)$ disk assuming a gas-to-dust ratio of 100. $M_{\text {int }}$ is the mass of the interior layer. $M_{\text {surf }}$ is the mass of the surface layer. $\mathrm{H}_{2}$ emission arises only from the optically thin molecular gas in surface layer of the disk (Carmona et al. 2008).

energy distribution (SED) of prototypical Herbig Ae/Be stars (Chiang et al. 2001), we computed the expected amount of gas in the interior and surface layer as function of the temperature for disks of mass $0.02 \mathrm{M}_{\odot}$ and $0.11 \mathrm{M}_{\odot}$ (see Fig. 2). The mass limits derived from the $\mathrm{H}_{2} 0-0 \mathrm{~S}(1)$ and $0-0 \mathrm{~S}(2)$ line observations are smaller than the amount of warm gas in the interior layer, but much larger than the amount of molecular gas in the surface layer. Fig. 2 shows that the amount of gas in the surface layer is very small $\left(<10^{-2} \mathrm{M}_{J} \sim 3 \mathrm{M}_{\oplus}\right)$ and almost independent of the total mass of the disk. If the two-layer model is an adequate representation of the structure of the disk, the thermal flux levels of $\mathrm{H}_{2}$ mid-infrared emission are below the detection limit of the observations, because the mass of $\mathrm{H}_{2}$ in the surface layer is very small.

In Carmona et al. (2008), based on a CG97 two-layer model, assuming LTE thermal emission, we calculated the $\mathrm{H}_{2}$ mid-IR fluxes expected from optically thick Herbig Ae disks (see Sect. 5.2 of Carmona et al. 2008 for a detailed description of the calculation). We found that for a source distant $140 \mathrm{pc}$, the expected fluxes are of the order of $10^{-16}$ erg s $\mathrm{s}^{-1} \mathrm{~cm}^{-2}$ for the $0-0 \mathrm{~S}(1)$ line and $10^{-17} \mathrm{erg} \mathrm{s}^{-1} \mathrm{~cm}^{-2}$ for the $0-0 \mathrm{~S}(2)$ line, and line-to continuum flux ratios of $<10^{-3}$. These line flux levels are two orders of magnitude below the sensitivity limits of our observations $\left(5 \times 10^{-15} \mathrm{erg} \mathrm{s}^{-1} \mathrm{~cm}^{-2}\right)$. If the two-layer approximation to the structure of the disk is correct, we are essentially "blind" to most of the warm $\mathrm{H}_{2}$ in the disk because it is located in the optically thick interior layer of the disk.

Nevertheless, some detections of mid-IR $\mathrm{H}_{2}$ emission from disks have been reported in the Herbig Ae/Be stars AB Aur (Bitner et al. 2007) and HD 97048 (Martin-Zaïdi et al. 2007), and in the T Tauri stars Sz 102, EC 74, EC 82, Ced 110 IRS6, EC 92, and ISO-Cha 237 (Lahuis et al. 2007). An interesting question to address is the reason for the high $\mathrm{H}_{2}$ fluxes observed in these sources. A possibility is that an additional mechanism (X-rays, UV heating, e.g., Glassgold et al. 2007; Nomura et al. 2007) heats the molecular gas in the surface layer making $\mathrm{T}_{\text {gas surf }}>\mathrm{T}_{\text {dust surf }}$. An additional scenario is to invoke a gas-to-dust ratio much larger than the canonical value of 100 in the surface layer of the disk (e.g., as a result of dust coagulation and sedimentation). In order to explore the influence of a change in the gas-to-dust ratio and the thermal decoupling of gas 
and dust in the surface layer, in Carmona et al. (2008) we calculated the expected 0-0 $\mathrm{S}(0)$ to $0-0 \mathrm{~S}(4) \mathrm{H}_{2}$ line fluxes from a $\mathrm{M}_{\mathrm{DISK}} 0.01 \mathrm{M}_{\odot}$ disk model, as a function of the surface gas-to-dust ratio (ranging from 100 to 14000 ) for $\mathrm{T}_{\text {gas surf }} / \mathrm{T}_{\text {dust surf }}$ ranging from 1.0 to 2.0. We obtained that detectable $0-0 \mathrm{~S}(1)$ and $0-0 \mathrm{~S}(2) \mathrm{H}_{2}$ line flux levels can be achieved if $\mathrm{T}_{\text {gas surf }} / \mathrm{T}_{\text {dust surf }}>2$ and if the gas-to-dust ratio in the surface layer is greater than 1000. $\mathrm{H}_{2}$ emission levels are very sensitive to departures from the thermal coupling between the molecular gas and dust in the surface layer. Our results suggest that in the observed sources the molecular gas and the dust in the surface layer have not significantly departed from thermal coupling and that the gas-to-dust ratio in the surface layer is very likely lower than 1000. A definitive interpretation of our results awaits the development of future, more sophisticated models.

\section{Searching for near-IR $\mathrm{H}_{2}$ emission from protoplanetary disks with CRIRES \\ 3.1. Motivation.}

To probe the gas in the terrestrial planet forming region of the disk. $\mathrm{H}_{2}$ near-IR lines probe hot gas at $T \sim 1000-3000 \mathrm{~K}$. This gas is located from a tenth of AU up to a few AU.

\subsection{Previous work.}

The $v=1-0 \mathrm{~S}(1) \mathrm{H}_{2}$ line at $2.2218 \mu \mathrm{m}$ has been detected in few classical $\mathrm{T}$ Tauri stars (CTTS): TW Hya, GG Tau A, LkCa 15 (Weintraub et al. 2000, Bary et al. 2002, 2003), AA Tau, CW Tau, UY Aur, GM Tau (Shukla et al. 2003), CS Cha (Weintraub et al. 2005), ECHAJ0843.3-7905 (Howat \& Greaves 2007) and $\mathrm{LkH} \alpha 264$ (Itoh et al. 2003, Carmona et al. 2007), and in four weak-line T Tauri stars (WTTS): DoAr 21 (Bary et al. 2003), V773 Tau (Shukla et al. 2003), Sz33 and Sz 41 (Weintraub et al. 2005).

\subsection{Observations \& Data Reduction.}

We observed the classical T Tauri star $\mathrm{LkH} \alpha 264$ and the debris disk 49 Cet, with the ESO-VLT cryogenic high-resolution $\left(R \sim 45000,6.6 \mathrm{~km} \mathrm{~s}^{-1}\right)$ infrared echelle spectrograph CRIRES (Käufl et al. 2004), mounted on ESO UT1 "Antu" 8-m telescope atop Cerro Paranal Chile, during the CRIRES science-verification phase (November 8 - 9, 2006). We employed the wave-ID $27 / 1 / \mathrm{n}$ and the wave-ID $25 /-1 / \mathrm{n}$, providing a spectral coverage from 2.0871 to $2.1339 \mu \mathrm{m}$ and from 2.2002 to $2.2552 \mu \mathrm{m}$ respectively. The observations were performed using a $46^{\prime \prime}$ long, $0.4^{\prime \prime}$ wide, north-south oriented slit, nodding the telescope $10^{\prime \prime}$ along the slit. A random jitter smaller than $2^{\prime \prime}$ was added to the telescope in addition to the nodding offset at each nodding position to correct for bad pixels and decrease systematics due to the detector. The total integration time was of $720 \mathrm{~s}$ for LkH $\alpha 264$ and of 240s for 49 Cet. Spectrophotometric standard stars at similar airmass to the science target were observed immediately following the science observations for performing the telluric correction. We searched for $v=1-0 \mathrm{~S}(1) \mathrm{H}_{2}$ emission at 2.1218 $\mu \mathrm{m}, v=1-0 \mathrm{~S}(0) \mathrm{H}_{2}$ emission at $2.2233 \mu \mathrm{m}$ and $v=2-1 \mathrm{~S}(1) \mathrm{H}_{2}$ emission at 2.2477 $\mu \mathrm{m}$. The data was reduced using the CRIRES pipeline and the ESO/CPL recipes. To correct for telluric absorption and flux-calibrate the science spectrum, the one-dimensional science spectrum obtained was divided by the one-dimensional spectrum of the standard star (for further details on the data reduction procedure, telluric correction and flux calibration see Sect. 2.1 of Carmona et al. 2007)

\subsection{Results.}

Our observations confirm the previous detections of the $\mathrm{H}_{2}$ 1-0 S(1) line reported by Itoh et al. (2003). However, in contrast to Itoh et al. (2003), the $\mathrm{H}_{2} 1-0 \mathrm{~S}(0)$ line is detected in our CRIRES spectra of $\mathrm{LkH} \alpha 264$. Our CRIRES observation show, for the first time, the simultaneous detection of the 1-0 S(1) and 1-0 S(0) $\mathrm{H}_{2}$ line from a protoplanetary disk. 

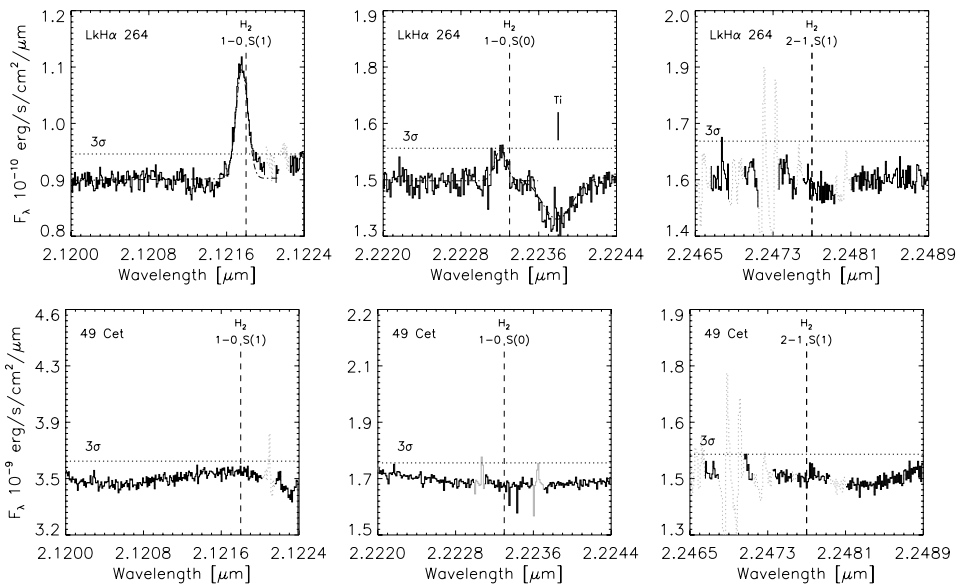

Figure 3. CRIRES spectra of $\mathrm{LkH} \alpha 264$ (upper panels) and 49 Cet (lower panels) in the regions of the $\mathrm{H}_{2} v=1-0 \mathrm{~S}(1), \mathrm{H}_{2} \quad v=1-0 \mathrm{~S}(0)$ and $\mathrm{H}_{2} \quad v=2-1 \mathrm{~S}(1)$ emission lines. The $\mathrm{H}_{2} \quad v=1-0 \mathrm{~S}(1)$ and the $\mathrm{H}_{2} v=1-0 \mathrm{~S}(0)$ lines are detected in $\mathrm{LkH} \alpha 264$. Photospheric Ti features at 2.2217 (not shown) and $2.2238 \mu \mathrm{m}$ (central upper panel) are observed in LkH $\alpha 264$. The Gaussian fits to the detected lines are illustrated in dash-dot lines. The $\mathrm{H}_{2} v=2-1 \mathrm{~S}(1)$ line is not present in $\mathrm{LkH} \alpha$ 264. In the case of 49 Cet none of the three $\mathrm{H}_{2}$ features are present in emission or absorption. Horizontal dotted lines show the $3 \sigma$ continuum flux limits. The spectra are not corrected for $\mathrm{V}_{\mathrm{LSR}}$ of the star. Regions of poor telluric correction are in gray-dotted lines in the spectra (Carmona et al. 2007).

Table 2. $\mathrm{H}_{2}$ near-IR emission line fluxes and upper limits

\begin{tabular}{|c|c|c|c|c|c|}
\hline Star & $\mathrm{H}_{2}$ line & $\begin{array}{c}\lambda \\
{[\mu \mathrm{m}]}\end{array}$ & {$\left[\times 10^{-10}\right.$} & $\begin{array}{l}\text { continuum } \\
\left.\operatorname{ergs~s}^{-1} \mathrm{~cm}^{-2} \mu \mathrm{m}^{-1}\right]\end{array}$ & {$\left[\begin{array}{c}\text { line flux } \\
{\left[\times 10^{-14} \operatorname{ergs~s}^{-1} \mathrm{~cm}^{-2}\right]}\end{array}\right.$} \\
\hline \multirow[t]{3}{*}{$\mathrm{LkH} \alpha 264$} & $1-0 \mathrm{~S}(1)$ & 2.1218 & & $0.9(0.04)$ & 0.30 \\
\hline & $1-0 \mathrm{~S}(0)$ & 2.2233 & & $1.5(0.08)$ & 0.10 \\
\hline & $2-1 \mathrm{~S}(1)$ & 2.2477 & & $1.6(0.10)$ & $<0.05$ \\
\hline \multirow[t]{3}{*}{$49 \mathrm{Cet}$} & $1-0 \mathrm{~S}(1)$ & 2.1218 & & $35(1.0)$ & $<0.5$ \\
\hline & $1-0 \mathrm{~S}(0)$ & 2.2233 & & $17(1.8)$ & $<0.9$ \\
\hline & $2-1 \mathrm{~S}(1)$ & 2.2477 & & $15(3.1)$ & $<1.6$ \\
\hline
\end{tabular}

Notes: ${ }^{a}$ Upper limits calculated using a line $F W H M$ of $6.6 \mathrm{~km} \mathrm{~s}^{-1}$.

The $\mathrm{H}_{2} 2-1 \mathrm{~S}(1)$ line is not seen in $\mathrm{LkH} \alpha 264$. The central wavelength of the 1-0 S(1) $\mathrm{H}_{2}$ emission in $\mathrm{LkH} \alpha 264$ was measured to be $2.121757 \pm 0.000005 \mu \mathrm{m}$. This corresponds to a velocity shift of $-5.6 \pm 1.0 \mathrm{~km} \mathrm{~s}^{-1}$ (taking into account Earth's velocity at the time of observation), a velocity coincident with the rest velocity of the star $(-5.9 \pm 1.2$ $\mathrm{km} \mathrm{s}^{-1}$, Itoh et al. 2003). The $\mathrm{H}_{2} 1-0 \mathrm{~S}(0)$ feature at $2.2233 \mu \mathrm{m}$ is detected with a $3 \sigma$ level confidence. Employing a Gaussian fit, the central wavelength of the line found is $2.22321 \pm 0.00005 \mu \mathrm{m}$. This corresponds to a velocity shift of $-12 \pm 7 \mathrm{~km} \mathrm{~s}^{-1}$ which is in agreement with the velocity shift found in the 1-0 S(1) line. The $F W H M$ of the lines are $20.6 \pm 1 \mathrm{~km} \mathrm{~s}^{-1}$ for the $\mathrm{H}_{2} 1-0 \mathrm{~S}(1)$ line and $19.8 \pm 1 \mathrm{~km} \mathrm{~s}^{-1}$ for the $\mathrm{H}_{2} 1-0 \mathrm{~S}(0)$ line. The measured line fluxes are $3.0 \times 10^{-15}$ and $1.0 \times 10^{-15} \mathrm{erg} \mathrm{s}^{-1} \mathrm{~cm}^{-2}$ for the $\mathrm{H}_{2}$ 1-0 $\mathrm{S}(1)$ and $\mathrm{H}_{2}$ 1-0 $\mathrm{S}(0)$ respectively. The 2-1 $\mathrm{S}(1) \mathrm{H}_{2}$ line is not observed in $\mathrm{LkH} \alpha$ 264. Assuming a $F W H M$ of $6.6 \mathrm{~km} \mathrm{~s}^{-1}$ (CRIRES resolution), a $3 \sigma$ flux upper limit of $5.3 \times 10^{-16} \mathrm{ergs} \mathrm{s}^{-1} \mathrm{~cm}^{-2}$ is derived for the line. Assuming an error of $20 \%$ in the flux calibration of the spectra, the $1-0 \mathrm{~S}(0) / 1-0 \mathrm{~S}(1)$ line ratio in $\mathrm{LkH} \alpha 264$ is $0.33 \pm 0.1$ and the 2-1 $\mathrm{S}(1) / 1-0 \mathrm{~S}(1)$ line ratio is $<0.2$. These line ratios are consistent with the line ratios of a gas at LTE at a temperature cooler than $1500 \mathrm{~K}$ (Mouri 1994).

In the case of 49 Cet none of the three $\mathrm{H}_{2}$ features are present in emission or absorption. A summary of the $3 \sigma$ flux upper limits is presented in Table 2. 


\subsection{Discussion.}

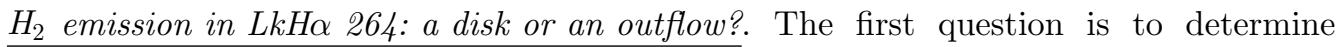
whether the $\mathrm{H}_{2}$ emission observed in $\mathrm{LkH} \alpha 264$ originates in an outflow (shock excited emission) or in a disk. The small velocity shift, the line shape (well reproduced by a disk model), and the fact that the emission is spatially unresolved are not in favor of shock excited $\mathrm{H}_{2}$. An additional strong argument against shock excitation of $\mathrm{H}_{2}$ is that LkHa 264 does not exhibit [OI] forbidden emission at $6300 \AA$ (Cohen and Kuhi 1979); a classical signature of outflows in T Tauri stars. The lack of this line indicates that in $\mathrm{LkH} \alpha 264$ the outflow is not present or at least that it is very weak. We conclude that the $\mathrm{H}_{2}$ emission observed in $\mathrm{LkH} \alpha 264$ originates very likely in a disk.

Excitation mechanism of the $\mathrm{H}_{2}$ emission in $\mathrm{LkH \alpha}$ 264. Thermal and non-thermal excitation mechanisms are distinguishable on the basis of line ratios (Mouri 1994 and references there in). With Figure 3b of Mouri (1994), we find that the measured 1-0 S(0)/1-0 $\mathrm{S}(1)(0.33 \pm 0.1)$ and the $2-1 \mathrm{~S}(1) / 1-0 \mathrm{~S}(1)(<0.2)$ line ratios in $\mathrm{LkH} \alpha 264$ are consistent with thermal emission of a gas cooler than $1500 \mathrm{~K}$ thermally excited by UV photons (i.e., UV photons NOT in the Lyman-Werner band at 912-1108 $\AA$ ). Assuming a gaussian error distribution, given the line 1-0 $\mathrm{S}(0) / 1-0 \mathrm{~S}(1)$ ratio measured, the probability that the heating mechanism is X-ray excitation is less than $1 \%$.

$\mathrm{H}_{2}$ emitting region and inclination of the $\mathrm{LkH \alpha} 264$ disk. The spectral resolution of CRIRES $\left(\approx 6.6 \mathrm{~km} \mathrm{~s}^{-1}\right)$ and the thermal width of a $1500 \mathrm{~K}$ line $\left(\approx 2.4 \mathrm{~km} \mathrm{~s}^{-1}\right)$ are significantly smaller than the $F W H M$ of $20 \mathrm{~km} \mathrm{~s}^{-1}$ of the $\mathrm{H}_{2}$ lines observed in $\mathrm{LkH} \alpha 264$. Therefore, the line width must be linked to the dynamics of the gas in the region that is emitting the line. Implementing the two-layer Chiang and Goldreich (1997) disk model code CG plus (Dullemond et al. 2001), we modeled the disk around $\mathrm{LkH} \alpha 264$, and found that the regions of the disk with a surface layer at $T_{s}<1500 \mathrm{~K}$ are located at $R>0.1$ AU. Modeling of the single peaked 1-0 S(1) line shape (for the details of the model see Carmona et al. 2007) indicates that the disk is close to face-on $\left(i<35^{\circ}\right)$. The best model fit suggests that the disk of $\mathrm{LkH} \alpha 264$ is inclined $20^{\circ}$ for a $\mathrm{H}_{2}$ emitting region extending from 0.1 to $10 \mathrm{AU}$ with a power law relation of the intensity as a function of radius with exponent $\alpha=-2$. If the $1-0 \mathrm{H}_{2} \mathrm{~S}(1)$ line intensity decreases with an exponent $\alpha=-2$ as a function of radius, then $50 \%$ of the line flux is produced within $0.1 \mathrm{AU}$ and $1 \mathrm{AU}$ of the $\mathrm{LkH} \alpha 264 \mathrm{disk}, 40 \%$ of the line flux is emitted within 1 and $7 \mathrm{AU}$ and the rest of the flux at larger radii.

Mass of optically thin hot $\mathrm{H}_{2}$ in LkHa 264 and 49 Cet. Using a similar equation to Eq. 2.1 from the 1-0 S(1) $\mathrm{H}_{2}$ line flux in $\mathrm{LkH} \alpha 264$ and the upper limit to the flux of the same line in 49 Cet, assuming optically thin gas in LTE, we calculated the mass $\mathrm{H}_{2}$ gas at $1500 \mathrm{~K}$ in both sources. We found that there are a few lunar masses of hot $\mathrm{H}_{2}$ gas in the inner disk of $\mathrm{LkH} \alpha 264$ and less than a tenth of a lunar mass of hot $\mathrm{H}_{2}$ in the inner disk of 49 Cet. The lack of $\mathrm{H}_{2}$ ro-vibrational emission in the spectra of 49 Cet, combined with non detection of pure rotational lines of $\mathrm{H}_{2}$ (Chen et al. 2006) and the absence of $\mathrm{H} \alpha$ emission suggest that the gas in the inner disk of 49 Cet has dissipated. These results together with the previous detection of ${ }^{12} \mathrm{CO}$ emission at sub-mm wavelengths (Zuckerman et al. 1995; Dent et al. 2005) point out that the disk of 49 Cet should have a large inner hole, and it is strongly suggestive of theoretical scenarios in which the disk disappears inside-out. This could be due to inside-out photoevaporation, or to the presence of an unseen low-mass companion(s).

Detections and non detections of near-IR $\mathrm{H}_{2}$ emission from T Tauri stars. Carmona et al. (2007) we present a detailed comparative analysis of the physical properties of classical $\mathrm{T}$ Tauri stars in which the $\mathrm{H}_{2}$ 1-0 S(1) line has been detected versus 
non-detected. We found that there is a higher chance of observing the $\mathrm{H}_{2}$ near-infrared lines in CTTS with a high $U-V$ excess and a strong $\mathrm{H} \alpha$ line. This result suggests that there is a higher probability of detecting the $\mathrm{H}_{2}$ 1-0 S(1) line in systems with high accretion. In contrast to weak-lined T Tauri stars, there is no apparent correlation between the X-ray luminosity and the detectability of the $\mathrm{H}_{2}$ 1-0 S(1) line in classical T Tauri stars. Taken as a group, $\mathrm{LkH} \alpha 264$ and the CTTS in which near-IR $\mathrm{H}_{2}$ emission has been detected exhibit typical properties of classical $\mathrm{T}$ Tauri stars. Therefore, we expect near-IR ro-vibrational $\mathrm{H}_{2}$ lines from $\mathrm{T}$ Tauri disks to be detected on a routine basis in the near future.

\section{Conclusions}

The advent of infrared high-resolution spectrographs mounted on large aperture telescopes allow for the first time the study of the gaseous component of the disk in the planet forming region $(\mathrm{R}<50 \mathrm{AU})$. Here, we presented two efforts for constraining the properties of the molecular hydrogen in a sample of Herbig Ae/Be, T Tauri stars and a debris disk.

In the first project, we searched for fundamental emission of $\mathrm{H}_{2}$ at 17 and 12 micron employing VISIR, ESO's mid-infrared high resolution spectrograph. Our aim was to probe the warm gas $(T \sim 150-1000 \mathrm{~K})$ in the giant planet forming region of the disk. None of the sources show fundamental $\mathrm{H}_{2}$ emission. From the upper limits on the line fluxes derived, we estimated that there is less than a tenth of Jupiter mass of optically thin $\mathrm{H}_{2}$ at $T=150 \mathrm{~K}$ and less than a few Earth mass at higher temperatures. These results are consistent with the two-layer disk model (CG 97). In this model only the molecular gas in the optically thin surface layer of the disk emits the $\mathrm{H}_{2}$ lines. Typical Herbig Ae disks have a very small mass of molecular gas $\left(<10^{-2} \mathrm{M}_{J}\right)$ in the surface layer. We calculated the expected $\mathrm{H}_{2}$ fluxes assuming a gas in LTE and a gas-to-dust ratio of 100. We found that the line fluxes are of the order of $10^{-16}$ to $10^{-17} \mathrm{erg} \mathrm{s}^{-1} \mathrm{~cm}^{-2}$, two orders of magnitude fainter than our detection limits. If the two-layer model is correct we are "blind" to the $\mathrm{H}_{2}$ gas in the interior layer. This result explains the numerous nondetections of mid-IR $\mathrm{H}_{2}$ emission towards several pre-main sequence stars with disks. The few detections reported can be explained as the result of a thermal decoupling of the gas and the dust (gas hotter than the dust) or a change in gas-to-dust ratio in the surface layer. Our non-detections suggest that in our sources the $\mathrm{H}_{2}$ and dust in the surface layer have not significantly departed from thermal coupling $\left(\mathrm{T}_{\text {gas }} / \mathrm{T}_{\text {dust }}<2\right)$ and the gas-to-dust ratio in the surface layer is very likely $<1000$.

In the second project, we searched for near-infrared ro-vibrational $\mathrm{H}_{2}$ emission from LkH $\alpha 264$ a classical T Tauri star and 49 Cet a debris disk with detections of CO emission in the sub-mm. Our aim was to probe the hot gas $(T \sim 1000 \mathrm{~K})$ present in the terrestrial planet region of the disks. We detected the 1-0 S(1) and 1-0 $\mathrm{S}(0) \mathrm{H}_{2}$ lines in $\mathrm{LkH} \alpha 264$ and derived an upper limit to the $2-1 \mathrm{~S}(1) \mathrm{H}_{2}$ line. Given that the velocity of the lines is coincident with the velocity of $\mathrm{LkH} \alpha 264$, that both lines have a similar FWHM of 20 $\mathrm{km} \mathrm{s}^{-1}$ (well reproduced by a disk model) and that $\mathrm{LkH} \alpha 264$ does not have evidence for outflows, we concluded that the observed emission very likely arises from a circumstellar disk. This is the first time that the 1-0 S(1) and 1-0 $\mathrm{S}(0) \mathrm{H}_{2}$ lines are detected in a protoplanetary disk. From the line ratios, we deduced that the $\mathrm{H}_{2}$ gas emitting the line is at temperature lower than $1500 \mathrm{~K}$ and that the gas is likely thermally excited by UV photons. Modeling of the disk around $\mathrm{LkH} \alpha 264$ and the shape of the $1-0 \mathrm{~S}(1) \mathrm{H}_{2}$ line suggests that the disk is observed close to face on $\left(i<35^{\circ}\right)$ and that most of the near-IR $\mathrm{H}_{2}$ emission is produced at less than 1 AU. The flux of the 1-0 $\mathrm{S}(1) \mathrm{H}_{2}$ line indicates that there is a few lunar masses of hot $(T \sim 1500 \mathrm{~K})$ optically thin $\mathrm{H}_{2}$ in the inner disk 
( $\mathrm{R} \sim 0.1 \mathrm{AU})$ of $\mathrm{LkH} \alpha 264$. In the case of 49 Cet none of the three $\mathrm{H}_{2}$ near-IR lines are observed in the spectra. The flux upper limits on the 1-0 $\mathrm{S}(1) \mathrm{H}_{2}$ line indicate that there is less than a fraction of lunar mass in the inner $(\mathrm{R}<1 \mathrm{AU})$ disk of 49 Cet. This result combined with the absence of near-IR excess in the spectral energy distribution, the lack of $\mathrm{H} \alpha$ in emission and the non-detection of mid-IR $\mathrm{H}_{2}$ emission (from Spitzer), suggest that the inner disk of 49 Cet has a hole in the gas and in the dust.

These projects show how high-resolution infrared spectroscopy is a useful tool for constraining the structure of protoplanetary disks, even in the case non-detections. Future studies, in larger samples are essential to identify statistical trends between the gas and dust properties of the disk as a function of the age and multiplicity.

\section{References}

Bary, J. S., Weintraub, D. A., \& Kastner, J. H. 2002, ApJL, 576, L73

Bary, J. S., Weintraub, D. A., \& Kastner, J. H. 2003, ApJL, 586, 1136

Bitner, M. A., Richter, M. J., Lacy, J. H., Greathouse, T. K., Jaffe, D. T., \& Blake, G. A. 2007, ApJL, 661, L69

Carmona, A., et al. 2008, A\&A, 477, 839

Carmona, A., van den Ancker, M. E., Henning, T., Goto, M., Fedele, D., \& Stecklum, B. 2007, $A \mathscr{E} A, 476,853$

Carr, J. S. 2005, in High Resolution Infrared Spectroscopy in Astronomy, Edited by H.U. Kufl, R. Siebenmorgen, and A.F.M. Moorwood. Springer-Verlag Berlin/Heidelberg, 2005, p. 203.

Chen, C. H., et al. 2006, ApJS, 166, 35

Chiang, E. I. \& Goldreich, P. 1997, ApJ , 490, 368

Chiang, E. I., Joung, M. K., Creech-Eakman, M. J., Qi, C., Kessler, J. E., Blake, G. A., \& van Dishoeck, E. F. 2001, ApJ, 547, 1077

Cohen, M., \& Kuhi, L. V. 1979, ApJS, 41, 743

Dent, W. R. F., Greaves, J. S., \& Coulson, I. M. 2005, MNRAS, 359, 663

Dullemond, C. P., Dominik, C., \& Natta, A. 2001, ApJ, 560, 957

Glassgold, A. E., Najita, J. R., \& Igea, J. 2007, ApJ, 656, 515

Hollenbach, D., et al. 2005, ApJ, 631, 1180

Howat, S. K. R., \& Greaves, J. S. 2007, MNRAS, 379, 1658

Itoh, Y., Sugitani, K., Ogura, K., \& Tamura, M. 2003, PASJ, 55, L77

Käuf, H. U. et al. 2004, SPIE, 5492, 1218

Lagage, P. O. et al. 2004, The Messenger, 117, 12.

Lahuis, F., van Dishoeck, E. F., Blake, G. A., Evans, N. J., II, Kessler-Silacci, J. E., \& Pontoppidan, K. M. 2007, ApJ, 665, 492

Lundin, L. K. VLT VISIR Pipeline User Manual. VLT-MAN-ESO-19500-3852. 2006

Martin-Zaïdi, C., Lagage, P.-O., Pantin, E., \& Habart, E. 2007, ApJL, 666, L117

Mouri, H. 1994, ApJ, 427, 777

Najita, J. R., Carr, J. S., Glassgold, A. E., \& Valenti, J. A. 2007, in Protostars and Planets, V, Edited by B. Reipurth, D. Jewitt, and K. Keil, University of Arizona Press, Tucson, 2007, p. $507-522$

Nomura, H., Aikawa, Y., Tsujimoto, M., Nakagawa, Y., \& Millar, T. J. 2007, ApJ, 661, 334

Pascucci, I., et al. 2006, ApJ, 651, 1177

Richter, M. J., Jaffe, D. T., Blake, G. A., \& Lacy, J. H. 2002, ApJL, 572, L161

Sako, S., Yamashita, T., Kataza, H., Miyata, T., Okamoto, Y. K., Honda, M., Fujiyoshi, T., \& Onaka, T. 2005, ApJ, 620, 347

Sheret, I., Ramsay Howat, S. K., \& Dent, W. R. F. 2003, MNRAS, 343, L65

Shukla, S. J., Bary, J. S., Weintraub, D. A., \& Kastner, J. H. 2003, Bulletin of the American Astronomical Society, 35, 1209

Thi, W. F., et al. 2001, ApJ, 561, 1074

Weintraub, D. A., Kastner, J. H., \& Bary, J. S. 2000, ApJ, 541, 767

Weintraub, D. A., Bary, J. S., Kastner, J. H., Shukla, S. J., \& Chynoweth, K. 2005, Bulletin of the American Astronomical Society, 37, 1165

Zuckerman, B., Forveille, T., \& Kastner, J.H. 1995, Nature, 373, 494 